\title{
Neural correlates of appetitive-aversive interactions in Pavlovian fear conditioning
}

\author{
Helen M. Nasser and Gavan P. McNally ${ }^{1}$ \\ School of Psychology, The University of New South Wales, Sydney, NSW 2052, Australia
}

\begin{abstract}
We used Pavlovian counterconditioning in rats to identify the neural mechanisms for appetitive-aversive motivational interactions. In Stage I, rats were trained on conditioned stimulus (CS)-food (unconditioned stimulus [US]) pairings. In Stage II, this appetitive CS was transformed into a fear CS via pairings with footshock. The development of fear responses was retarded in rats that had received Stage I appetitive training. This counterconditioning was associated with increased levels of phosphorylated mitogen activated protein kinase immunoreactivity (pMAPK-IR) in several brain regions, including midline thalamus, rostral agranular insular cortex (RAIC), lateral amygdala, and nucleus accumbens core and shell, but decreased expression in the ventrolateral quadrant of the midbrain periaqueductal gray. These brain regions showing differential pMAPK-IR have previously been identified as part of the fear prediction error circuit. We then examined the causal role of RAIC MAPK in fear learning and showed that Stage II fear learning was prevented by RAIC infusions of the MEK inhibitor PD098059 ( $0.5 \mu \mathrm{g} /$ hemisphere). Taken together, these results show that there are opponent interactions between the appetitive and aversive motivational systems during fear learning and that the transformation of a reward CS into a fear CS is linked to heightened activity in the fear prediction error circuit.
\end{abstract}

Mammals, including humans, are faced with two broad problems for survival. They must identify, learn about, and respond appropriately to cues in the environment which signal events critical for survival, such as food, water, and sexual partners. They must also identify, learn about, and respond appropriately to cues in the environment which signal threats to survival, such as predators and the absence of food or water. These problems are competing. Animals must rapidly switch their learning and behavior from satisfying the demands of one motivational system to satisfying the demands of the other. For example, an animal foraging for food and water must switch its behavioral repertoire when it encounters a predator or finds that a food source has been depleted. Such competition is pervasive. Animal and human decision making requires assignment of value to stimuli and events as appetitive, and hence to be sought, or aversive, and hence to be avoided. However, this value assignment must be flexible and sensitive to changing environmental contingencies.

To solve for these problems, it has been argued that mammals possess an appetitive motivational system that mediates approach and reward and an aversive motivational system that mediates defense and fear, with recruitment of these systems controlled via associative learning (Amsel 1962; Konorski 1967; Rescorla and Solomon 1967; Dickinson and Dearing 1979). The aversive and appetitive motivational systems are mutually inhibitory. A signal for danger not only arouses the aversive system to elicit defensive behavior but also inhibits the appetitive motivational system to suppress behaviors such as foraging, eating, and drinking. For example, during Pavlovian appetitive conditioning, a rat is subjected to pairings of a CS with a food US. The consequence of these pairings is that rats exhibit orienting responses to the CS upon subsequent presentations as well as behaviors directed toward the magazine where food was delivered (Holland 1977). If that CS is then arranged to signal an aversive US, such as footshock, the appetitive conditioned responses (CRs) are replaced with defensive or fear responses (Bolles and Fanselow

\footnotetext{
${ }^{1}$ Corresponding author

E-mail g.mcnally@unsw.edu.au

Article is online at http://www.learnmem.org/cgi/doi/10.1101/Im.029744.112.
}

1980). For example, the rat ceases to display magazine entries and begins to express the species-specific defense response of freezing. The rate at which such fear is acquired is slower, or retarded, relative to a CS that has not undergone prior appetitive training (Bouton and Peck 1992; Nasser and McNally 2012). The prior appetitive training interferes with the later fear learning.

Whereas much is known about the neural substrates for appetitive learning and fear learning separately (Maren and Quirk 2004; Pickens and Holland 2004; Rodrigues et al. 2004), little is known about the neural substrates for their interaction. There is evidence from nonhuman primates that lateral habenula (LHb) neurons may contribute to these interactions. For example, during Pavlovian conditioning, the responses of LHb neurons to a CS signaling an aversive unconditioned stimulus (US) are opposite to those observed to a CS signaling an appetitive US (Matsumoto and Hikosaka 2009a,b). If a neuron is excited by an aversive CS it is inhibited by an appetitive CS or vice versa. In the $\mathrm{LHb}$, individual neurons respond in the same manner (excitation) to a CS signaling an aversive US as to a CS signaling the absence of an appetitive US (Matsumoto and Hikosaka 2009a). The activity of these neurons is consistent with the argument that appetitive and aversive systems are mutually inhibitory (Konorski 1967; Dickinson and Dearing 1979). Furthermore, electrophysiological recordings in the primate amygdala have identified neurons preferentially excited by delivery of reward or punishment and suppressed by the opposite US (Belova et al. 2007, 2008). Interestingly these neurons also demonstrate suppression by the omission of the preferred value (Belova et al. 2007; Herry et al. 2008) consistent with the proposal that the omission of an aversive event is equivalent to the delivery of an appetitive one and vice versa. However, the causal role of activity changes in these or other brain regions during appetitive-aversive interactions is unknown.

The aims of these experiments were to assess motivational interactions during fear learning, identify the potential neural correlates of such interactions, and identify the causal role of these correlates in these interactions. To this end we used a counterconditioning task. Rats were first trained in appetitive conditioning whereby a CS signaled delivery of a food US. We then transformed 
that appetitive CS into a fear CS by arranging that it now signaled footshock US. Across the experiments we measured expression of appetitive and fear CRs, used immunohistochemistry to identify brain regions recruited during counterconditioning, and used site-specific microinjections to study their role in this counterconditioning.

\section{Experiments la and $\mathrm{lb}$}

Experiment 1a involved a single-factor, two-group design. In Stage I, group Paired received Pavlovian appetitive conditioning so that an auditory CS signaled delivery of a food pellet US to a magazine. In Stage II this same CS then signaled a footshock US. A control group received the same apparatus exposure as group Paired during Stage I but did not receive appetitive training. This group then received the same Stage II fear conditioning as group Paired. We (Nasser and McNally 2012) and others (Bouton and Peck 1992) have reported that under these conditions fear learning in group Paired is retarded relative to that in group Control and that such retardation is specifically due to the association of the CS with the appetitive US formed during Stage I (Nasser and McNally 2012).

Experiment $1 \mathrm{~b}$ employed a single-factor, three-group design as seen in Table 1. In Stage I, group Paired received Pavlovian appetitive conditioning whereby an auditory CS signaled delivery of a food pellet US to a magazine. In Stage II this same CS was now arranged to signal a footshock US. Group Reward received the same Stage I training and was simply exposed to the apparatus during Stage II. Group Fear was simply exposed to the apparatus during Stage I and then received Stage II training. Rats were sacrificed after the first day of Stage II training, and their brains removed and processed for immunohistochemical detection of phosphorylated extracellular regulated kinase (ERK)/mitogenactivated protein kinase (pMAPK). ERK/MAPK couples signaling via the protein kinase $A$ and protein kinase $C$ pathways with cyclic AMP response element binding protein phosphorylation (Roberson et al. 1999; Adams and Sweatt 2002). ERK/MAPK signaling plays an important role in synaptic plasticity and associative learning. Fear conditioning increases expression of pMAPK in amygdala and other brain regions, and amygdala infusions of inhibitors of upstream MAPK signaling, such as MAPK kinase (MEK), and impairs long-term potentiation and long term memory for fear conditioning (Schafe et al. 2001, 2002; Rodrigues et al. 2004). As such, pMAPK expression is a useful learning-related marker of neural activity and plasticity.

\section{Results and Discussion}

\section{Experiment la}

The top panel of Figure 1 shows the mean ( \pm SEM) levels of magazine entries across Stage I appetitive training for group Paired. There was a significant increase in magazine entries across days of acquisition, $F_{(1,7)}=32.5, P<0.05$.

The bottom panels of Figure 1 show the mean and SEM levels of magazine entries (left panel), head-jerks (middle panel), and freezing (right panel) across the $2 \mathrm{~d}$ of Stage II training for groups

Table 1. Summary of design, Experiment 1

\begin{tabular}{|c|c|c|}
\hline & Stage I & Stage II \\
\hline $\begin{array}{l}\text { Paired } \\
\text { Fear }\end{array}$ & $T+$ food & $\begin{array}{l}T+\text { shock } \\
T+\text { shock }\end{array}$ \\
\hline Reward & $T+$ food & \\
\hline
\end{tabular}

(T) Tone CS, (+food) two sugar pellets, (+shock) 0.5-mA 0.5-sec footshock.

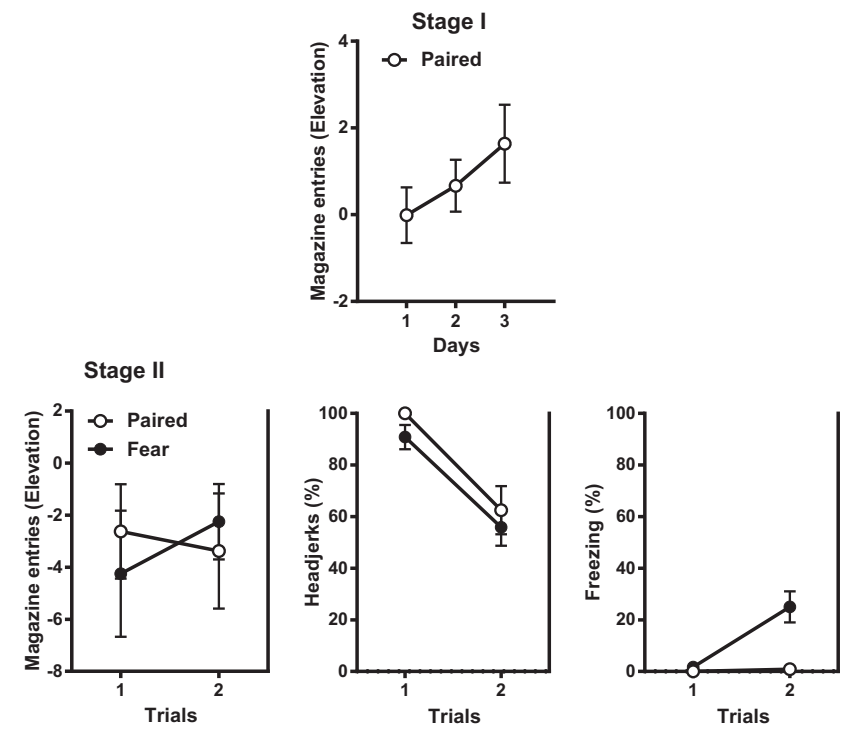

Figure 1. Experiment 1a. Mean ( \pm SEM) elevation scores for magazine entries during Stage I (top). Mean ( \pm SEM) elevation scores for magazine entries (bottom left), head-jerking (bottom middle), and freezing (bottom right) during the CS in Stage II.

Paired and Fear. There were no significant differences in pre-CS magazine entries between group Paired $(M=6, \mathrm{SEM}=1.6)$ and group Fear $(M=4, \mathrm{SEM}=1.5), F_{(1,14)}<1, P>0.05$. There were also no significant differences between groups or across the $2 \mathrm{~d}$ of Stage II training, $F^{\prime} \mathrm{S}_{(1,14)}<1, P>0.05$, in magazine entries. It is worth noting that the low magazine elevation score for group Paired in Experiment 1a reflects a high level of magazine pre-CS responding. Further analysis revealed that CS magazine entries were significantly higher for group Paired $(M=4, \mathrm{SEM}=0.7)$ relative to group Fear $(M=1.1, \mathrm{SEM}=0.7), F_{(1,14)}=10.8, P<0.05$, on the first day of Stage II. During Stage II, group Paired $(M=15 \%$, $\mathrm{SEM}=4.1$ ) showed significantly higher levels of pre-CS head-jerks compared to group Fear $(M=4 \%, \mathrm{SEM}=1.3), F_{(1,14)}=6.3, P<$ 0.05 . There were no significant overall differences between groups in CS-elicited head-jerking during Stage II, $F^{\prime} \mathrm{s}_{(1,14)}=2.0, P>0.05$. There was a significant linear decrease in head-jerks across the two Stage II trials, $F_{(1,14)}=26.3, P<0.05$, and this did not differ between groups, $F_{(1,14)}<1, P<0.05$. There was no pre-CS freezing in either group across the $2 \mathrm{~d}$ of Stage II training (group Paired $M=0 \%, \mathrm{SEM}=0$; group Fear $M=0 \%, \mathrm{SEM}=0), F_{(1,14)}<1, P>$ 0.05 . Overall there was significantly less freezing in group Paired vs. group Fear, $F_{(1,14)}=24.1, P<0.05$, a significant linear increase in freezing across trials, $F_{(1,14)}=11.3, P<0.05$, and a significant difference between groups in this increase, $F_{(1,14)}=9.8, P<0.05$. These results indicate that fear acquisition was retarded to a CS with a history of appetitive training compared to a neutral CS.

\section{Experiment $1 \mathrm{~b}$}

Stage I appetitive training proceeded uneventfully for groups Paired and Reward. There was a significant increase in magazine entries across days of acquisition, $F_{(1,10)}=22.8, P<0.05$, and no differences between groups, $F_{(1,10)}=2, P>0.05$. Nor was there a significant group $\times$ day interaction, $F_{(1,10)}=2.3, P>0.05$. There was no freezing during the first CS-footshock US. Rats were sacrificed after the first Stage II CS-footshock US pairing and pMAPK immunoreactivity (pMAPK-IR) was studied in several brain regions linked to fear learning, including the prefrontal cortex, amygdala, midline thalamus, nucleus accumbens, hypothalamus, 
A

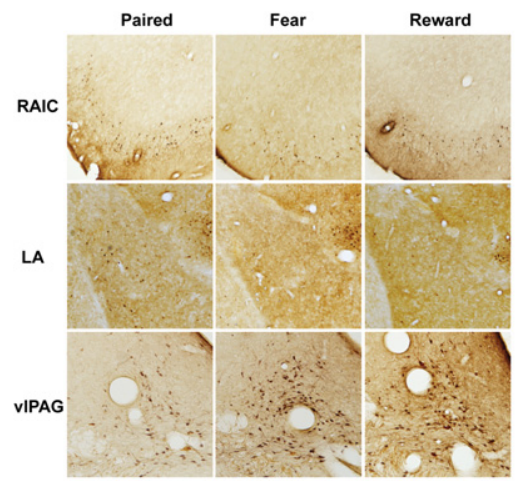

B
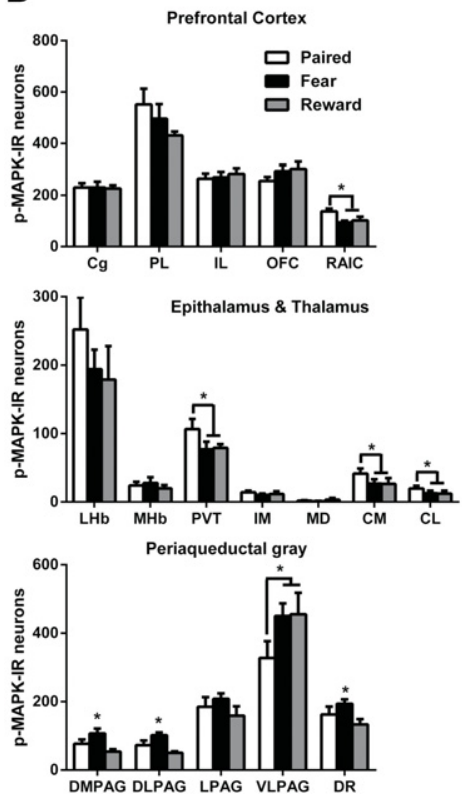
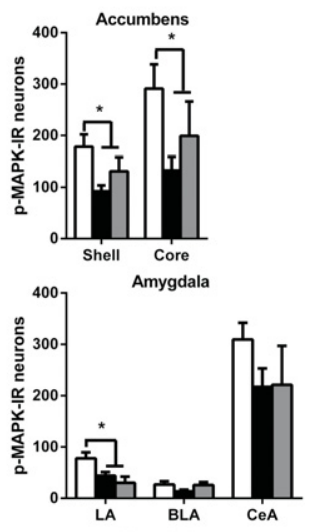

Hypothalamus

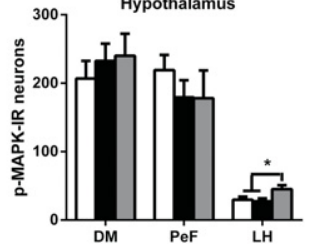

Figure 2. Experiment $1 \mathrm{~b}$. (A) Representative photomicrographs of pMAPK-immunoreactivity (pMAPK-IR) in the rostral agranular insular cortex (RAIC), lateral amygdala (LA), ventrolateral periaqueductal gray (vIPAG) for groups Paired, Fear, and Reward. (B) Mean and SEM counts of pMAPK-IR in the prefrontal cortex, nucleus accumbens, epithalamus, midline and intralaminar thalamus, amygdala, PAG, and hypothalamus for groups Paired, Fear, and Reward. (*) $P<0.05$.

and periaqueductal gray (PAG) (McNally et al. 2011); the results are shown in Figure 2. Stage II behavioral data were not recorded due to technical difficulties with recording equipment. In several brain regions there was a similar profile of between group differences in pMAPK-IR. In the paraventricular thalamus (PVT), caudal centromedial thalamus $(\mathrm{CM})$, centrolateral thalamus $(\mathrm{CL})$, rostral agranular insular cortex (RAIC), lateral amygdala (LA), and nucleus accumbens shell (AcbSh) and core (AcbC) there were significant differences between groups in pMAPK-IR so that in each region group Paired showed significantly greater pMAPK-IR relative to groups Fear and Reward, $F^{\prime} \mathrm{s}_{(1,17)}=4.5$ for PVT, 5.6 for CM, 8.8 for CL, 5.0 for RAIC, 4.8 for LA, 6.9 for AcbSh, 5.5 for AcbC; all $P<0.05$. In each of these regions, groups Fear and Reward did not differ from each other, all $F^{\prime} \mathrm{s}_{(1,17)}<1.7, P>0.05$. There were no other differences between groups in other areas of the prefrontal cortex, amygdala, midline thalamus, or epithalamus $F^{\prime} \mathrm{s}_{(1,17)}<1.5, P>0.05$.

In the PAG, there were different profiles of pMAPK-IR dependent on the PAG column. In the dorsomedial (dmPAG), dorsolat-

eral (dlPAG), and dorsal raphe (DR) pMAPK-IR was greatest in group Fear compared with groups Paired and Reward, $F^{\prime} \mathrm{s}_{(1,17)}=$ $4.8,9$, and 4.5 , respectively, all $P<0.05$, and there were no differences between groups Paired and Reward, $F^{\prime} \mathrm{s}_{(1,17)}<1, P>0.08$. However, in the ventrolateral PAG (vlPAG) pMAPK-IR was significantly reduced in group Paired compared with groups Fear and Reward, $F_{(1,17)}=4.9, P<0.05$. There were no other significant differences in pMAPK-IR between groups in PAG, $F_{(1,17)}<$ $1.5, P>0.05$.

In the hypothalamus there were significant differences between groups in the lateral hypothalamus (LH). Here, pMAPK-IR was greatest in group Reward compared with groups Paired and Fear, $F_{(1,17)}=5.7, P<0.05$, which did not differ from each other, $F_{(1,17)}<1, P>0.05$. There were no significant differences between groups in pMAPK-IR for the dorsomedial (DM) and perifornical (PeF) hypothalamus, $F^{\prime} \mathrm{s}_{(1,17)}<1, P>0.05$.

\section{Experiment 2}

Experiments 1a and $1 \mathrm{~b}$ showed that a fear CS was retarded in the rate at which it was transformed into an appetitive CS and that this counterconditioning was accompanied by increased pMAPK-IR in a number of brain regions, including the RAIC, midline thalamus, and LA. However, the role of increased pMAPK-IR in fear learning as well as appetitive to aversive counterconditioning is not well understood. Thus, whereas MAPK signaling in the LA has been linked to both LA synaptic plasticity and the acquisition of Pavlovian fear conditioning (Schafe et al. 2002), the role of MAPK in these other regions during fear learning or counterconditioning is unclear. The RAIC is especially interesting in this regard. The RAIC projects extensively to the LA and these projections are important for influencing LA function (Jasmin et al. 2003). However, the role of MAPK signaling in the RAIC during Pavlovian fear conditioning and appetitive-aversive interactions is unknown.

The aim of this experiment was to study the role of MAPK in the RAIC during fear learning as a result of appetitive to aversive counterconditioning. Rats were implanted with bilateral guide cannulas aimed at the RAIC. This experiment used a between groups $2 \times 2$ factorial design, where the first factor referred to the presence of Stage I appetitive conditioning (groups Paired) or not (groups Fear) and the second factor refers to the type of infusion into the RAIC prior to Stage II fear conditioning (the MEK inhibitor PD098059 [0.5 $\mu \mathrm{g} /$ hemisphere] vs. the vehicle dimethyl sulfoxide [DMSO]).

\section{Results and Discussion}

\section{Histology}

Figure 3 shows the location of the microinjection cannula tips in the RAIC. Nine rats were excluded from the analyses due to misplaced cannulas. Thus the final group sizes were: groups PD Paired, $n=10$, PD Fear, $n=10$, DMSO Paired, $n=10$, and DMSO Fear, $n=9$.

\section{Behavior}

Figure 4 shows the mean $( \pm$ SEM) behavioral performances. During Stage I, there was a significant linear increase in magazine entries during the auditory CS, $F_{(1,17)}=31.3, P<0.05$. There was no overall difference between groups (PD Paired and DMSO Paired), $\quad F_{(1,17)}=2.4, \quad P>0.05$, or group $\times$ day interaction, $F_{(1,17)}<1, P>0.05$. Therefore groups PD Paired and DMSO Paired acquired similar levels of appetitive conditioning to the CS. 


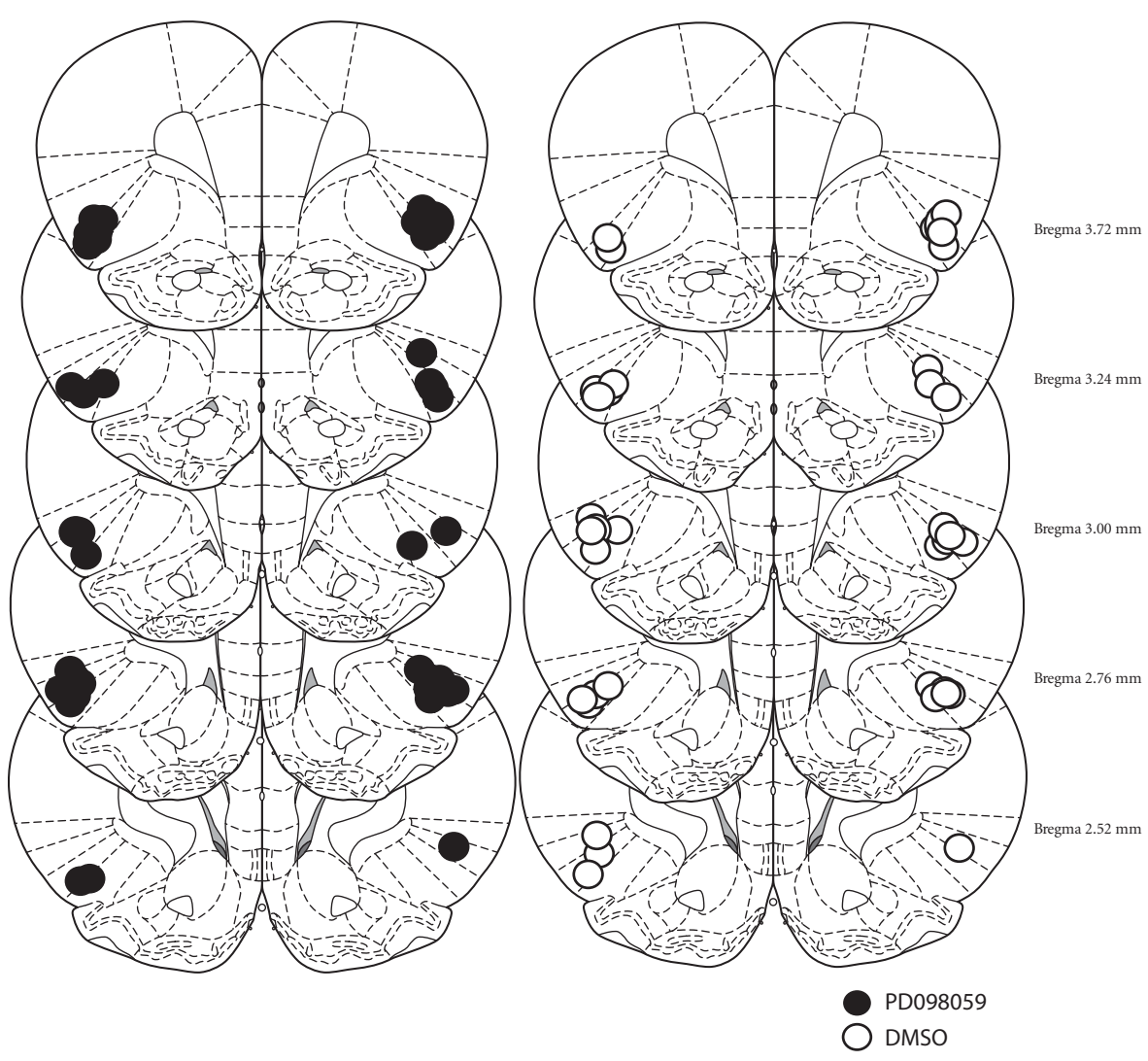

Figure 3. Location of microinjection cannula tips in rostral agranular insular cortex (RAIC) for rats receiving infusion of DMSO (white circles) or PD098059 (black circles). (Adapted from Paxinos and Watson 2007 and reprinted with permission from Elsevier (C) 2007.)

Overall pre-CS magazine entries during Stage II were low; however, there were significantly higher levels of magazine entries for groups Paired compared to groups Control regardless of RAIC infusion type, PD Paired $(M=1.7, S E M=0.6)$, DMSO Paired $(M=1$, SEM $=0.5)$, PD Fear $(M=0.2$, SEM $=0.1)$, and DMSO Fear $(M=0.2, \operatorname{SEM}=0.2), F_{(1,35)}=8.4, P<0.05$. There were no other significant differences between groups, $F_{(1,35)}<1$, $P>0.05$. There was a significant effect of prior appetitive training, so that groups Paired showed higher levels of magazine entries compared to Fear controls, $F_{(1,35)}=5.8, P<0.05$. Overall there was a decrease in magazine entries across Stage II for all groups, $F_{(1,35)}=6.8, P<0.05$. There was no overall effect of drug or drug $\times$ training interaction, $F_{(1,35)}<2, P>0.05$. There were no other significant interactions across training, $F_{(1,35)}<$ $1.3, P>0.05$.

Stage II pre-CS head-jerking was low and there was no effect of drug or training condition, PD Paired $(M=2.8 \%, \mathrm{SEM}=1.4)$, DMSO Paired $(M=7.2 \%, \mathrm{SEM}=2.5)$, PD Fear $(M=3.3 \%, \mathrm{SEM}=$ $1.4)$, and DMSO Fear $(M=0.7 \%$, $\mid \mathrm{SEM}=0.4), F^{\prime} \mathrm{s}_{(1,35)}<3.8, P>$ 0.05 . There was a significant effect of prior appetitive training, so that groups Paired showed higher levels of head-jerking compared to groups Control, $F_{(1,35)}=11.8, P<0.05$. Overall there was a significant decrease in head-jerking across Stage II for all groups, $F_{(1,35)}=35.3, P<0.05$. There was no overall effect of drug or drug $\times$ training interaction, $F_{(1,35)}<1, P>0.05$.

Stage II pre-CS freezing was low and there was no effect of drug or training on pre-CS freezing in Stage II, PD Paired $(M=$ $8.5 \%$, SEM $=3.4)$, DMSO Paired $(M=22.2 \%$, SEM $=4)$, PD Fear $(M=23.8 \%, \operatorname{SEM}=5.4)$, and DMSO Fear $(M=28.3 \%, \mid \mathrm{SEM}=$
8.6), $F^{\prime} \mathrm{s}_{(1,35)}<3.4, P>0.05$. There was a significant effect of prior appetitive training, so that groups Paired showed lower levels of freezing compared to groups Fear, $F_{(1,35)}=4.7, \quad P<0.05$. Overall there was an increase in freezing across Stage II for all groups, $F_{(1,35)}=$ $37.5, P<0.05$. There was also a significant main effect of RAIC infusion so that PD groups showed lower levels of freezing than those receiving DMSO, $F_{(1,35)}=4.4, \quad P<0.05$. Inspection of the figure suggests that PD reduced freezing most in group Paired, however there was no significant drug $\times$ training interaction, $F_{(1,35)}<1, P>0.05$. Nonetheless, group PD Paired showed significantly less freezing than the remaining groups $F_{(1,35)}=8, P<0.05$, and there was no significant difference between PD Fear and DMSO fear, $F_{(1,35)}<1, P>0.05$. This suggests that the RAIC infusions of the MEK inhibitor PD098059 impaired the acquisition of fear in appetitive to aversive counterconditioning.

\section{Discussion}

Here we studied appetitive-aversive interactions during Pavlovian fear conditioning. Rats were initially trained in an appetitive conditioning phase involving pairings of an auditory CS with a food pellet US. As a result, rats expressed Pavlovian appetitive CRs to the CS. Then in a second stage, this appetitive CS was transformed into a fear CS by arranging that it signaled an aversive footshock. Across the course of Stage II training, expression of appetitive CRs was reduced and replaced with the expression of a fear CR (freezing). The rate at which the aversive CR emerged during this counterconditioning was retarded relative to a control group that did not receive Stage I appetitive conditioning. This finding shows retardation of fear learning during appetitive to aversive counterconditioning.

Although there were several differences among the groups in terms of their prior training, the retardation of fear learning observed here is linked specifically to the prior appetitive training of the target CS. For example, in Experiment 1a, group Paired had received 36 prior presentations of the CS and the US and it is possible that such presentations could retard fear learning independently of any appetitive conditioning to the CS, for example via learned inattention to the CS because it was a good predictor of its consequences (i.e., Pearce-Hall negative transfer), or because the CS exposures themselves yielded some latent inhibition, or because the food pellet supported conditioning of the accompanying contextual cues. We have previously empirically assessed these alternate explanations. For example, Nasser and McNally (2012) demonstrated that 36 presentations of either a CS alone or food US alone prior to aversive conditioning was insufficient to produce retardation of fear under exactly the same conditions as were used here. Thus, the retardation as demonstrated here is specific to the CS gaining appetitive properties in Stage I.

To identify the neural correlates of this appetitive-aversive interaction we assessed expression of pMAPK-IR in a variety of 

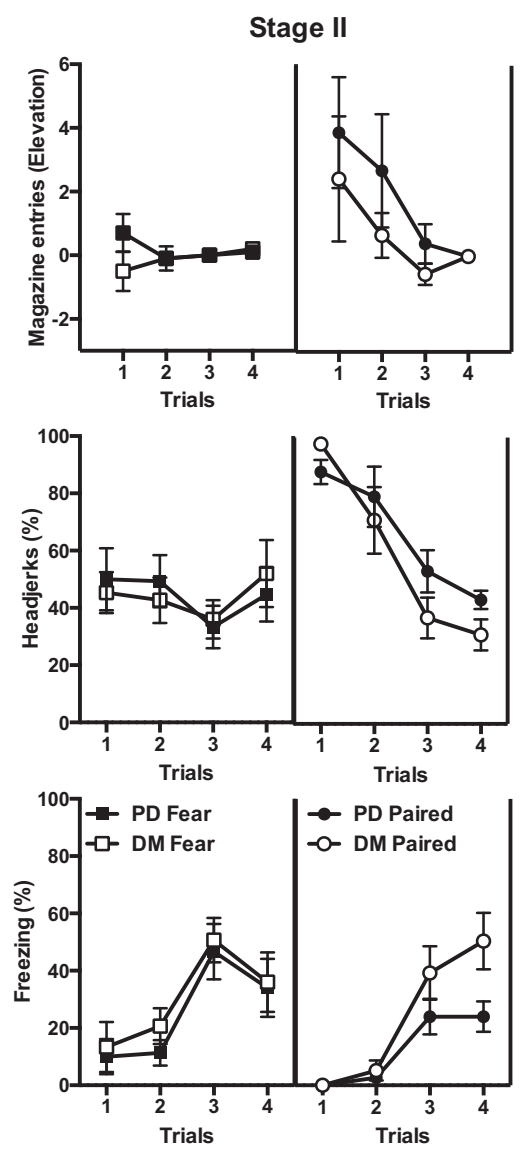

Figure 4. Experiment 2. Mean ( \pm SEM) elevation scores for magazine (top), and head-jerking (middle) and freezing (bottom) during the CS in Stage II. (PD) Infusion of PD098059, (DM) infusion of the vehicle DMSO.

brain regions linked to fear learning. These results showed that in the midline and intralaminar thalamus, RAIC, LA, and the nucleus accumbens, which included the AcbSh and AcbC, there was significantly increased pMAPK-IR during fear learning among animals undergoing appetitive to aversive counterconditioning compared to animals undergoing aversive conditioning or animals with just a history of appetitive conditioning. In contrast, in the ventrolateral quadrant of the PAG the differences were reversed so that there was significantly reduced PMAPK-IR during fear learning among animals undergoing fear conditioning after appetitive conditioning. The profile of pMAPK-IR in these brain regions is interesting because the vlPAG projects extensively to midline and intralaminar thalamus (Krout and Loewy 2000; Krout et al. 2002), which in turn project to the AcbSh, AcbC, as well as the RAIC (Berendse and Groenewegen 1991; Groenewegen and Berendse 1994; Wright and Groenewegen 1995; Vertes 2006; Vertes and Hoover 2008), and the RAIC, in turn, projects to the LA (Jasmin et al. 2003, 2004). This vlPAGmidline thalamus-prefrontal-amygdala circuit has been implicated in fear learning, and specifically in the coding of prediction error during fear learning in both rats and humans (Iordanova et al. 2006; Dunsmoor et al. 2008; Furlong et al. 2010; McNally et al. 2011).

The profile of group differences in pMAPK expression during appetitive to aversive counterconditioning may appear counterintuitive because MAPK has been linked to learning and synaptic plasticity, including fear learning (Roberson et al. 1999; Schafe et al. 2001, 2002; Adams and Sweatt 2002), yet group Paired that expressed the highest levels of pMAPK-IR in some regions, and the lowest in another, was significantly retarded in the development of fear learning during Stage II. However, this pattern of group differences in pMAPK-IR is consistent with an associative opponent motivational model of appetitive-aversive interactions (Konorski 1967; Dickinson and Pearce 1977; Dickinson and Dearing 1979). This model states that an appetitive CS will excite the appetitive motivational system and so inhibit the aversive motivational system. From this perspective, a Pavlovian appetitive CS is functionally equivalent to a conditioned inhibitor of fear. Thus, in the experiments here, Stage I appetitive training not only imbued the CS with positive associative strength in the appetitive system, but also with negative associative strength in the fear system. The significantly lower levels of pMAPK-IR in the vlPAG could reflect this aversive negative associative strength (McNally et al. 2011). In addition, during Stage II fear conditioning, this initially negative associative strength results in a larger positive prediction error for group Paired. This is because the discrepancy between actual (shock) and expected (reward or no shock) outcome of the Stage II fear conditioning trials was larger for the counterconditioning groups than the discrepancy between actual (shock) and expected (nothing) outcomes for the control group. The significantly higher levels of pMAPK-IR in the midline thalamus, Acb, RAIC, and LA could reflect this increased prediction error. This enhanced error was not detected behaviorally because the CS started Stage II with negative associative strength was slower to be transformed into a fear CS and so levels of freezing were significantly lower (Nasser and McNally 2012).

An alternate explanation for increased pMAPK-IR activity in these regions is the summation of neural populations that preferentially encode for reward or fear. Recent electrophysiological findings in monkeys have suggested that within the amygdala separable populations of neurons preferentially encode for the motivational value of a CS (Belova et al. 2007, 2008). Thus, because the Paired group received both reward and fear conditioning it is possible that increased pMAPK activity during counterconditioning reflects the summation of activity within these populations. Specifically, it could reflect the retrieval of the appetitive memory from Stage I and the formation of the fear memory in Stage II. In this regard it is worth noting that the Reward group did not receive presentation of the CS prior to sacrifice. Several studies suggest that the presentation of the cue after training can induce activation of the memory and increase kinase activity in the basolateral amygdala (BLA) and the Acb (for example, Szapiro et al. 2002; Shiflett et al. 2008). Although this is possible, it is unclear why this enhanced activation would also be seen in the thalamus and RAIC, for which there is little evidence for separable coding of appetitive and aversive motivation.

Consistent with a role for these regions in fear learning, we showed that RAIC infusions of the MEK inhibitor PD098059 prior to CS shock pairings in Stage II significantly impaired fear learning. This effect of PD098059 infusions cannot easily be attributed to nonspecific mnemonic or motoric effects because it had no effect on the expression of appetitive CRs. Instead, PD098059 infusions acted selectively to attenuate Stage II fear learning. The effect of PD098059 was significantly greater in the counterconditioning group. This lack of a significant interaction may have been due to insufficient statistical power and this possibility warrants further investigation. Regardless, this effect of MAPK inhibition is important because it shows a causal role for RAIC in fear learning during counterconditioning. The insular cortex was among the first cortical regions implicated in human fear learning via neuroimaging techniques (Buchel et al. 1998), and has also been implicated via protein imaging studies in rodents (Furlong et al. 2010). For example, Furlong et al. (2010) demonstrated 
that RAIC neurons were recruited by positive prediction error during fear learning. Yet the role of insular cortex in fear learning has remained unclear. Lesions of the posterior (i.e., parietal) insular cortex impair fear learning as measured by fear potentiated startle (Shi and Davis 1999) but not when measured via freezing (Lanuza et al. 2004), whereas lesions of the anterior insular, that encompassed the RAIC, significantly augment fear learning when assessed via freezing (Lacroix et al. 2000). More recently, lesions of the posterior insular region reduced the anxiety-relieving properties of a safety signal (Christianson et al. 2011, 2012).

In these experiments, the group differences in pMAPK-IR and also the effects of PD098059 infusion were largest during counterconditioning, as opposed to normal fear conditioning. This finding is not immediately consistent with a role for pMAPK in fear learning generally because such effects should also have been observed during normal fear conditioning (e.g., group Fear in Experiment 1b). This finding is not necessarily problematic. The parameters used here (US intensity and duration, number of pairings per day) were selected because we have shown them to be optimal for detection of counterconditioning (Nasser and McNally 2012). Thus, a different set of parameters may have yielded larger effects during normal fear conditioning.

The only other brain regions that showed group differences in these experiments were the hypothalamus, dorsal PAG, and DR. In the dorsal PAG and DR, there was increased pMAPK-IR for group Fear compared to groups Paired and Reward. Thus, this increase may reflect delivery of the shock US and/or pairing of the CS and shock US related to fear learning that was attenuated in group Paired. In LH, pMAPK-IR was significantly higher in group Reward than in the remaining groups. LH is critical for appetitive motivation including food seeking (Kelley et al. 2005) and pMAPK-IR there may reflect the positive associative strength of the appetitive CS. However, this was not present in group Paired undergoing appetitive to aversive counterconditioning. This is difficult to interpret, but one possibility is that it reflects a role for inhibition of the LH in suppression of appetitively motivated behaviors by the fear system. Interestingly, there was no evidence for significant differences between groups in PMAPK-IR in the LHb. The firing of neurons in the primate habenula have been shown to be sensitive to appetitive-aversive interactions (Matsumoto and Hikosaka 2007, 2009a; Hong et al. 2011), and have provided an important model for understanding these interactions at a cellular level. The reason(s) for this lack of differences in LHb pMAPK-IR is unclear. One possibility is that LHb, by virtue of its inhibition of midbrain dopamine neurons (Ji and Shepard 2007), is critical for inhibition of the appetitive motivational system by the aversive motivational system, but not for appetitive inhibition of the aversive motivational system studied here. A second possibility is that MAPK does not play a critical role in these learning induced changes in habenula neuronal firing. Hence, a different marker may yield different findings. However, these possibilities are speculative and the role of habenula in motivational interactions during fear learning warrants further investigation.

In conclusion, we used pMAPK immunohistochemistry as well as infusions of the MEK inhibitor PD098059 to study the neural correlates of appetitive-aversive motivational interactions during Pavlovian fear conditioning. We identified several brain regions, including vlPAG, midline thalamus, Acb, RAIC, and LA, that were sensitive to these interactions as evidenced by differential expression of pMAPK-IR during appetitive to aversive counterconditioning. vlPAG reflected the negative associative strength of the CS, whereas midline thalamus, Acb, RAIC, and LA reflected the augmented positive prediction error during this counterconditioning. We also showed that RAIC pMAPK was causal to fear learning during counterconditioning because fear learning was prevented by RAIC infusions of the MEK inhibitor PD098059.
Taken together, the findings reported here support the claim that there are opponent interactions between the appetitive and aversive motivational systems during Pavlovian fear conditioning and show that the transformation of a reward CS into a fear CS is linked to heightened activity in the fear prediction error circuit.

\section{Materials and Methods}

\section{Subjects}

Subjects were experimentally naive male Wistar rats (300-400 g) obtained from a commercial supplier (Monash Animal Services, Gippsland, Victoria, Australia). After arrival, rats were housed in groups of eight in plastic cages and maintained on a 12-h lightdark cycle (lights on at 7:00AM). Food and water were available ad libitum until 2 d before behavioral training, after which rats were allowed 1-h access to food following daily training sessions, with unrestricted access to water. The procedures used were approved by the Animal Care and Ethics Committee at the University of New South Wales and were conducted in accordance with the National Institutes of Health (NIH) "Guide for the Care and Use of Laboratory Animals" (NIH Publications No. 80-23) revised 1996. The procedures were designed to minimize the number of animals used.

\section{Behavioral apparatus}

For all experiments, training was conducted in eight identical experimental chambers $(24 \mathrm{~cm}$ [length] $\times 30 \mathrm{~cm}$ [width] $\times 21 \mathrm{~cm}$ [height]; Med Associates Inc.); the roof, rear wall, and hinged door were constructed of clear Perspex, and the end walls were made of stainless steel. A recessed magazine (3 $\mathrm{cm}$ in diameter) was located behind a $4-\mathrm{cm} \times 4-\mathrm{cm}$ opening in the center of one end wall. Infrared photocells detected entries into each magazine entry. Sugar pellets were delivered to the magazine through an external automated food hopper. Each chamber was enclosed in light and sound attenuating cabinets $(55.9 \mathrm{~cm}$ [length] $\times 35.6$ $\mathrm{cm}$ [width] $\times 38.1 \mathrm{~cm}$ [height]) fitted with fans for ventilation and low-level background noise. They contained a speaker for delivering an $85-\mathrm{dB} 1200-\mathrm{Hz}$ tone that acted as a CS.

To serve as distinct contexts, the chambers differed in their location (two separate rooms), visual (brightly lit vs. dark), tactile (Perspex vs. grid floors), and olfactory (rose oil vs. peppermint essence) properties. In context $A$ the set of chambers had a Perspex plate floor and were illuminated by a $28-\mathrm{V}$ house light. Each chamber stood $35 \mathrm{~mm}$ above a tray of corn cob bedding and dilute rose oil or dilute peppermint essence was placed in the bedding beneath these chambers. In context $B$ the chamber floors consisted of stainless steel rods, $4 \mathrm{~mm}$ in diameter, spaced $15 \mathrm{~mm}$ apart (center to center), that delivered a 0.6-mA scrambled footshock which was used as an aversive reinforcer. There was no illumination in these chambers. The odors in the chambers were fully counterbalanced. The tactile stimuli were not counterbalanced because the grid floor was needed to deliver the footshock. Digital video cameras were mounted on the rear wall of each box and connected to a digital multiplexer in the room that, in turn, was connected to a DVD recorder. All chambers were connected to a computer with Med-PC IV software (Med Associates) that controlled CS and US presentations and recorded the number of magazine entries.

\section{Surgery and histology}

In Experiment 2, before behavioral testing, rats were implanted with two unilateral guide cannulas directed bilaterally toward the RAIC. Rats were injected intraperitoneally with the anesthetic ketamine (concentration $100 \mathrm{mg} / \mathrm{kg}$; Ketapex, Apex Laboratories) and with $0.3 \mathrm{~mL} / \mathrm{kg}$ muscle relaxant xylazine (concentration $20 \mathrm{mg} / \mathrm{mL}$; Rompun, Bayer). They were shaved and then placed in a stereotaxic frame (Model 900, Kopf) with the incisor bar maintained at $\sim 3.3 \mathrm{~mm}$ below horizontal to achieve a flat skull position. Rats were implanted bilaterally with 26-gauge guide cannulas (Plastics One). Flat skull coordinates relative to bregma for the RAIC were $+2.7 \mathrm{~mm}$ anteroposterior (AP), $\pm 4.0-\mathrm{mm}$ 
mediolateral (ML), and $-5.0 \mathrm{~mm}$ dorso-ventral (DV). All guide cannulae were aimed $1 \mathrm{~mm}$ above target site with coordinates based on the rat brain atlas of Paxinos and Watson (2007). Cannulas were secured to the skull using jeweler's screws and acrylic cement. Obturators with dust caps were fitted to the guide cannulas to prevent occlusion. Immediately after surgery, rats were injected subcutaneously with $5 \mathrm{mg} / \mathrm{kg}$ carprofen and intramuscularly with $0.3 \mathrm{~mL}$ of procaine penicillin $(300 \mathrm{mg} / \mathrm{mL})$ and $0.3 \mathrm{~mL}$ of cefazolin $(100 \mathrm{mg} / \mathrm{mL})$. Rats were given 5 - to 7 -d postoperative recovery before the start of behavioral procedures, during which time they were monitored and weighed daily.

To confirm cannula placements, at the end of the experiment rats were overdosed with sodium pentobarbital and their brains removed. The brains were then sectioned coronally ( $40 \mu \mathrm{m}$ thick) using a cryostat at the level of the RAIC. Every second section was collected onto a glass slide, stained with cresyl violet, and coverslipped. Cannula placements were plotted onto Adobe illustrator templates (Paxinos and Watson 2007) with the use of a microscope. Rats with placements outside the RAIC were excluded from the analyses.

\section{Infusions}

For intracranial infusions, 33-gauge cannula injectors (which projected 1-mm ventral to the tip of the guide cannula) were connected to a $10-\mu$ L Hamilton syringe via polyethylene-20 tubing, which was mounted onto an infusion pump (KD Scientific). Rats received infusions of DMSO or a solution containing PD098059 (Tocris Bioscience), a specific inhibitor of the activation of MEK, an upstream regulator of MAPK, in 100\% DMSO $(1 \mu \mathrm{g} / \mu \mathrm{L})$ (Schafe et al. 1999). All microinjections were made in a volume of $0.5 \mu \mathrm{L}$ per side over $2 \mathrm{~min}$. After allowing an additional 2 min for diffusion, microinjectors were removed and rats were returned to their home cage for $20 \mathrm{~min}$, before being tested in the chamber.

\section{Immunohistochemistry}

Rats were returned to their home cage at the end of the 50-min test session. One hour after the commencement of test, rats were deeply anesthetized with sodium pentobarbital (100 mg/kg, i.p.) and transcardially perfused with sodium nitrite and heparin $(5000$ i.u. $/ \mathrm{mL}$ ) followed by paraformaldehyde (4\%) in phosphate buffer (PB), pH 7.4. Brains were then removed, postfixed (1 hr), and cytoprotected in sucrose overnight.

The entire forebrain and midbrain were sectioned using a cryostat (Leica Microsystems). Every fourth section was collected (40 $\mu \mathrm{m}$ thick, $160 \mu \mathrm{m}$ apart) to form a series which was stored in $0.1 \mathrm{M}$ PB saline (pH 7.2) containing $0.1 \%$ sodium azide. This was used to reveal pMAPK in combination with tyrosine hydroxylase $(\mathrm{TH})$ using two-color peroxidise immunohistochemistry. Free floating sections were washed repeatedly in $0.1 \mathrm{M} \mathrm{PB}$ ( $\mathrm{pH}$ 7.4) followed by two 30-min washes in 50\% ethanol, the second of which contained $3 \% \mathrm{H}_{2} \mathrm{O}_{2}$, and were then incubated in 5\% normal horse serum (NHS) in $\mathrm{PB}(\mathrm{pH} 7.4$ ) for $30 \mathrm{~min}$. Sections were then incubated in rabbit antiserum against pMAPK (1:2000; phosphor-p44-42 MAPK [THr202/Tyr204]; Cell Signaling Technology) and sheep antiserum TH (1:2000; Jackson ImmunoResearch Laboratories) for $48 \mathrm{~h}$ at room temperature with gentle agitation. The primary antibody was diluted in PBT-X comprising $0.1 \mathrm{M} \mathrm{PB}(\mathrm{pH} 7.4)$ containing $0.1 \%$ sodium azide, $2 \%$ NHS, and $0.2 \%$ Triton X-100. After washing off unbound primary antibody, sections were incubated overnight at room temperate in biotinylated donkey anti-rabbit IgG (1:2000; Jackson ImmunoResearch Laboratories) diluted in 2\% NHS PBT-X. After washing off unbound secondary antibody, sections were incubated for $2 \mathrm{hr}$ at room temperate in $\mathrm{ABC}$ reagent (Vector Elite kit $6 \mu \mathrm{L} / \mathrm{mL}$ avidin and $6 \mu \mathrm{L} / \mathrm{mL}$ biotin; Vector Laboratories). Black immunoreactive (IR) cytoplasm labeled for pMAPK was revealed by a nickel-intensified diaminobenzidine (DAB) reaction, with peroxide being generated by glucose oxidase. In this DAB reaction, sections were washed in $\mathrm{PB}$ ( $\mathrm{pH} 7.4$ ), followed by $0.1 \mathrm{M}$ acetate buffer ( $\mathrm{pH}$ 6.0), and then incubated for $15 \mathrm{~min}$ in $0.1 \mathrm{M}$ acetate buffer ( $\mathrm{pH}$ 6.0) containing $2 \%$ nickel sulfate, $0.025 \%$ $\mathrm{DAB}, 0.04 \%$ ammonium chloride, and $0.02 \% \mathrm{D}$-glucose. The peroxidise reaction was started by adding $0.2 \mu \mathrm{L} / \mathrm{mL}$ glucose oxidase and stopped using acetate buffer ( $\mathrm{pH}$ 6.0). Brain sections were then washed in $\mathrm{PB}(\mathrm{pH} 7.4)$ and processed again, in a similar manner, using biotinylated donkey anti-sheep IgC (1:1000; Jackson ImmunoResearch Laboratories) but without nickel-intensification to localize IR for $\mathrm{TH}$, revealed as a brown reaction product in order to easily identify specific brain regions. Sections were mounted onto gelatine-treated slides, dehydrated, cleared with histolene, and coverslipped with Entellan.

\section{Neuronal counting}

Manual counts of neurons IR for pMAPK were conducted through the rostro-caudal extent of each brain region, and analyzed by an observer unaware of each rat's group allocation. All sections counted were $160 \mu \mathrm{m}$ apart. pMAPK-IR neurons were counted using a transmitted light microscope at $10 \times 10$ magnification. Counts were made at the microscope across consecutive sections of the collected series on one side of the brain in a number of regions. Regions were delineated using clearly visible landmarks according to Paxinos and Watson (2007) unless otherwise stated. When landmarks did not clearly delineate regions, boundaries were created with a $10 \times 10$-grid reticule $(1 \mathrm{~mm} \times 1 \mathrm{~mm})$ located in the right eye piece of the microscope. Ten graduations of the grid were equivalent to $0.1 \mathrm{~mm}$ on the section. Labeled-IR was expressed as raw counts. IR counts in each region were summed across sections.

\section{PFC (4 bregma $3.64 \mathrm{~mm}$ to $3.00 \mathrm{~mm}$ )}

Medial. For the anterior part of the cingulated area, a grid $(0.1 \mathrm{~mm}$ $[\mathrm{h}] \times 0.05 \mathrm{~mm}[\mathrm{w}])$ was aligned with the DM edge of the section. For the prelimbic cortex, the grid was aligned with the dorsal aspect of the forceps minor (FMI) and a $0.12-\mathrm{mm}$ (h) area between the medial edge of the section and the FMI created. Similarly, for the infralimbic cortex (IL), a $0.08-\mathrm{mm}$ (h) area was created with the edge of the grid aligned at the point where the FMI and the edge of the section become parallel.

Lateral. For the orbital frontal cortex, the grid $(0.1 \mathrm{~mm} \times 0.1 \mathrm{~mm})$ was placed against the medial edge of a line extending $\sim 45^{\circ}$ from the dorsal tip of the FMI and placed to the dorsal of the rhinal fissure. For the RAIC, the grid was placed against the lateral edge of a line extending $\sim 45^{\circ}$ from the dorsal tip of the FMI and dorsally against the rhinal fissure.

\section{Acb (bregma $1.92 \mathrm{~mm}$ to $1.12 \mathrm{~mm}$ )}

The shell could be differentiated from the septum by the appearance of the tissue. The shell (AcbSh) and core (AcbC) were separated by a line extending from the lateral ventricle (LV), rounding the anterior commissure (AC) by half the width of the AC. The boundary of the AcbC (from dorsally located putamen) then continued half the AC laterally and dorsally to the AC reaching the LV.

\section{Amygdala (bregma $-2.4 \mathrm{~mm}$ to $-3.36 \mathrm{~mm}$ )}

The lateral part of the amygdala was restricted within the triangular shaped region of the external capsule. The LA was then separated from the BLA by a horizontal line through the center of the central amygdala.

\section{Thalamus and epithalamus (bregma $-2.90 \mathrm{~mm}$ to $-3.36 \mathrm{~mm}$ )}

Midline. The dense, dark labeling of the PVT nucleus and intermediodorsal nucleus (IMD) appeared distinct from the adjacent medial dorsal thalamus (MD). The PVT and the IMD were separated where the PVT narrows to meet the IMD. 
Intralaminar (bregma $-2.90 \mathrm{~mm}$ to $-3.36 \mathrm{~mm}$ )

The intralaminar nuclei formed a semicircular pattern bordering the MD. The caudal centromedian thalamic nucleus $(\mathrm{CM} ;-2.9$ $\mathrm{mm}$ and $-3.12 \mathrm{~mm}$ ) was restricted laterally by a vertical line through the lateral edge of the submedius thalamic nucleus (Sub). The CL nucleus was restricted medially by a vertical line through the lateral edge of the Sub.

Habenula (bregma $-2.40 \mathrm{~mm}$ to $-3.36 \mathrm{~mm}$ )

The MHb and LHb were restricted to the boundaries created by the stria medullaris and fascicular retroflexus.

\section{Hypothalamus (bregma -2.40 to $-3.36 \mathrm{~mm}$ )}

The dorsal part of the hypothalamus was restricted dorsally by the dorsal aspect of the third ventricle (3V), and ventrally by the ventral aspect of the fornix (F). The DM hypothalamus was formed by the area between the $3 \mathrm{~V}$ and medial edge of the mammillothalamic tract (MT), the PeF hypothalamus between the MT and lateral to the $\mathrm{F}$ (approximately half the width of the F lateral to $\mathrm{F}$ ), and the $\mathrm{LH}$, extending to the medial edge of the internal capsule.

\section{Mesencephalon: Periaqueductal gray (PAG) (bregma $-7.08 \mathrm{~mm}$ to $-8.20 \mathrm{~mm}$ )}

The caudal vlPAG was formed by the area bordered approximately by the mesencephalon 5 nucleus and trochlear nerve $(-7.68 \mathrm{~mm}$ and 8.20). The DR was separated from the vlPAG by a line extending $\sim 45^{\circ}$ from the ventral-lateral corner of the aqueduct (Aq).The lateral PAG and dIPAG were separated by a line extending $\sim 45^{\circ}$ from the dorsal-lateral corner of the Aq. The dlPAG and dmPAG were separated by a line extending perpendicularly, approximately half way along the dorsal-lateral edge of the Aq.

\section{Behavioral procedures}

\section{Magazine training}

On Day 1, all rats were exposed to the chambers in context A for a 20-min magazine training session. Twenty sugar pellets were delivered to the magazine at random intertrial intervals (ITI) with a mean of $120 \mathrm{sec}$ without any stimuli presentations. This training enabled the subjects to learn the location of the food US prior to Stage I. On Day 2, they were exposed to the chambers in room B in a 20 -min session with no food present. All subsequent sessions were conducted in context $\mathrm{B}$.

\section{Stage I}

Stage I training occurred on Days 3, 4, and 5 and rats were placed in the chambers for $\sim 50 \mathrm{~min}$. Rats were divided into three groups. For groups Paired and Reward, rats received 12 pairings of a 30 -sec tone with two $45-\mathrm{mg}$ sugar pellets delivered 0.5 sec apart. CS offset coincided with US onset. The ITI was variable, with an average of $240 \mathrm{sec}$. Group Fear received equal apparatus exposure without stimuli. Immediately following the last appetitive conditioning session, all rats received one 50-min session of box exposure. The rats in the paired condition received no stimuli, but the control rats received two presentations of the tone alone to reduce any unconditioned responding prior to the beginning of the next phase.

\section{Stage II. Experiment la}

Stage II training occurred on Days 6 and 7. For groups Paired and Fear aversive conditioning consisted of two 50-min sessions. Each session contained a single tone reinforced with a 1-sec 0.6-mA footshock immediately after the CS presentation. Tone onset occurred in the 5th minute of the session; extended exposure to the chambers was designed to reduce any fear to the apparatus.

\section{Stage II. Experiment $1 b$}

Stage II training involved $1 \mathrm{~d}$ and was identical to Day 6 for Experiment 1a for groups Paired and Fear. On this day, group Reward received equal context exposure without stimulus presentation

\section{Stage II. Experiment 2}

All behavioral procedures were exactly identical to those in Experiment 1a for groups Paired and Fear with the exception that there were $4 \mathrm{~d}$ of Stage II training immediately prior to each day rats were infused with either vehicle (DMSO) or PD098059 $(0.5 \mu \mathrm{g} /$ hemisphere $)$.

\section{Data analysis}

In all experiments, magazine entries (the number of times the rat entered their head into the magazine) and orienting responses to the CS were used as the primary measures of appetitive conditioning. These two forms of $\mathrm{CR}$ reflect learning about different features of the CS-US relation (so-called US-generated and CS-generated responses) (Rescorla and Holland 1982). To provide a measure of appetitive US-generated responding, the elevation of magazine entries was calculated by subtracting the number of magazine entries during the $30 \mathrm{sec}$ before the CS (pre-CS period) from the number of entries during the 30-sec CS. For CS-generated responding, behavior was scored every 2 sec as either head-jerking or not. Head-jerking was defined as the short rapid horizontal or vertical movements of the head usually oriented toward the food magazine or source of the audio output. Simultaneous display of head-jerk and hindquarter movement or rearing was scored as head-jerk (Holland 1977). For aversive learning, fear was scored every $2 \mathrm{sec}$ as either freezing or not freezing by two observers, one of whom was aware of group allocations and one unaware. Freezing was defined as the absence of all movement other than that required for breathing. The percentages of these observations scored as freezing by each observer were then calculated. The correlation between the two observer ratings exceeded 0.85 .

In all experiments, data were analyzed using planned orthogonal contrasts testing main effects and interactions with the type 1 error rate $(\alpha)$ controlled at 0.05 . For Experiment 1 a the degrees of freedom were $v 1=1$ and $v 2=7$; the $F$-critical for these analyses was 5.591 during Stage I and $v 1=1$ and $v 2=14$; the $F$-critical for these analyses was 4.6 during Stage II. Experiment $1 \mathrm{~b}$ had degrees of freedom set at $v 1=1$ and $v 2=10$; the $F$-critical for these analyses was 4.965 during Stage I, and degrees of freedom $v 1=1$ and $v 2=17$; the $F$-critical for these analyses was 4.451 for immunohistochemical analysis. In addition to this, in Experiment 2 the degrees of freedom were $v 1=1$ and $v 2=35$; the $F$-critical for these analyses was 4.121 . A multivariate approach to repeated measures data was taken (O’Brien and Kaiser 1985).

\section{Acknowledgments}

These experiments were supported by a Discovery Project grant from the Australian Research Council to G.P.M. (DP0877430). G.P.M. is an ARC QEII Fellow (DP0877430).

\section{References}

Adams JP, Sweatt JD. 2002. Molecular psychology: Roles for the ERK MAP kinase cascade in memory. Annu Rev Pharmacol Toxicol 42: 135-163.

Amsel A. 1962. Frustrative nonreward in partial reinforcement and discrimination learning: Some recent history and a theoretical extension. Psychol Rev 69: 306-328.

Belova MA, Paton JJ, Morrison SE, Salzman CD. 2007. Expectation modulates neural responses to pleasant and aversive stimuli in primate amygdala. Neuron 55: 970-984.

Belova MA, Paton JJ, Salzman CD. 2008. Moment-to-moment tracking of state value in the amygdala. J Neurosci 28: 10023-10030.

Berendse HW, Groenewegen HJ. 1991. Restricted cortical termination fields of the midline and intralaminar thalamic nuclei in the rat. Neuroscience 42: $73-102$. 
Bolles RC, Fanselow MS. 1980. A perceptual-defensive-recuperative model of fear and pain. Behav Brain Sci 3: 291-323.

Bouton ME, Peck CA. 1992. Spontaneous recovery in cross-motivational transfer counterconditioning. Anim Learn Behav 20: 313-321.

Buchel C, Morris J, Dolan RJ, Friston KJ. 1998. Brain systems mediating aversive conditioning: An event-related fMRI study. Neuron 20: 947-957.

Christianson JP, Jennings JH, Ragole T, Flyer JGN, Benison AM, Barth DS, Watkins LR, Maier SF. 2011. Safety signals mitigate the consequences of uncontrollable stress via a circuit involving the sensory insular cortex and bed nucleus of the stria terminalis. Biol Psychiatry 70: 458-464.

Christianson JP, Fernando ABP, Kazama AM, Jovanovic T, Ostroff LE, Sangha S. 2012. Inhibition of fear by learned safety signals: A mini-symposium review. I Neurosci 32: 14118-14124.

Dickinson A, Dearing MF. 1979. Appetitive-aversive interactions and inhibitory processes. In Mechanisms of learning and motivation: A memorial volume to Jerzy Konorksi (eds. A Dickinson, RA Boakes), pp. 203-231, Erlbaum, Totowa, NJ.

Dickinson A, Pearce JM. 1977. Inhibitory interactions between appetitive and aversive stimuli. Psychol Bull 84: 690-711.

Dunsmoor JE, Bandettini PA, Knight DC. 2008. Neural correlates of unconditioned response diminution during Pavlovian conditioning. NeuroImage 40: 811-817.

Furlong TM, Cole S, Hamlin AS, McNally GP. 2010. The role of prefrontal cortex in predictive fear learning. Behav Neurosci 124: 574-586.

Groenewegen HJ, Berendse HW. 1994. The specificity of the "nonspecific" midline and intralaminar thalamic nuclei. Trends Neurosci 17: 52-57.

Herry C, Ciocchi S, Senn V, Demmou L, Müller C, Lüthi A. 2008. Switching on and off fear by distinct neuronal circuits. Nature 454: 600-606.

Holland PC. 1977. Conditioned stimulus as a determinant of the form of the Pavlovian conditioned response. J Exp Psychol Anim Behav Process 3: 77-104.

Hong S, Jhou TC, Smith M, Saleem KS, Hikosaka O. 2011. Negative reward signals from the lateral habenula to dopamine neurons are mediated by rostromedial tegmental nucleus in primates. J Neurosci 31: $11457-11471$.

Iordanova MD, McNally GP, Westbrook RF. 2006. Opioid receptors in the nucleus accumbens regulate attentional learning in the blocking paradigm. J Neurosci 26: 4036-4045.

Jasmin L, Rabkin SD, Granato A, Boudah A, Ohara PT. 2003. Analgesia and hyperalgesia from GABA-mediated modulation of the cerebral cortex. Nature 424: $316-320$.

Jasmin L, Burkey AR, Granato A, Ohara PT. 2004. Rostral agranular insular cortex and pain areas of the central nervous system: A tract-tracing study in the rat. J Comp Neurol 468: 425-440.

Ji H, Shepard PD. 2007. Lateral habenula stimulation inhibits rat midbrain dopamine neurons through a GABA(A) receptor-mediated mechanism. J Neurosci 27: 6923-6930.

Kelley AE, Baldo BA, Pratt WE, Will MJ. 2005. Corticostriatalhypothalamic circuitry and food motivation: Integration of energy, action and reward. Physiol Behav 86: 773-795.

Konorski J. 1967. Integrative activity of the brain: an interdisciplinary approach. University of Chicago Press, Chicago, IL.

Krout KE, Loewy AD. 2000. Periaqueductal gray matter projections to midline and intralaminar thalamic nuclei of the rat. J Comp Neurol 424: $475-494$.

Krout KE, Belzer RE, Loewy AD. 2002. Brainstem projections to midline and intralaminar thalamic nuclei of the rat. J Comp Neurol 448: $53-101$.

Lacroix L, Spinelli S, Heidbreder CA, Feldon J. 2000. Differential role of the medial and lateral prefrontal cortices in fear and anxiety. Behav Neurosci 114: $1119-1130$.

Lanuza E, Nader K, Ledoux JE. 2004. Unconditioned stimulus pathways to the amygdala: Effects of posterior thalamic and cortical lesions on fear conditioning. Neuroscience 125: 305-315.
Maren S, Quirk GJ. 2004. Neuronal signalling of fear memory. Nat Rev Neurosci 5: 844-852.

Matsumoto M, Hikosaka O. 2007. Lateral habenula as a source of negative reward signals in dopamine neurons. Nature 447: 1111-1115.

Matsumoto M, Hikosaka O. 2009a. Representation of negative motivational value in the primate lateral habenula. Nat Neurosci 12: $77-84$.

Matsumoto M, Hikosaka O. 2009b. Two types of dopamine neuron distinctly convey positive and negative motivational signals. Nature 459: $837-841$.

McNally GP, Johansen JP, Blair HT. 2011. Placing prediction into the fear circuit. Trends Neurosci 34: 283-292.

Nasser HM, McNally GP. 2012. Appetitive-aversive interactions in Pavlovian fear conditioning. Behav Neurosci 126: 404-422.

O'Brien RG, Kaiser MK. 1985. The MANOVA approach for analyzing repeated measures designs: An extensive primer. Psychol Bull 97: 316-333.

Paxinos G, Watson C. 2007. The rat brain in stereotaxic coordinates, 6th ed. Elsevier Inc., Amsterdam, The Netherlands.

Pickens CL, Holland PC. 2004. Conditioning and cognition. Neurosci Biobehav Rev 28: 651-661.

Rescorla RA, Holland PC. 1982. Behavioral studies of associative learning in animals. Аппи Rev Psychol 33: 265-308.

Rescorla RA, Solomon RL. 1967. Two-process learning theory: Relationships between Pavlovian conditioning and instrumental conditioning. Psychol Rev 74: 151-182.

Roberson ED, English JD, Adams JP, Selcher JC, Kondratick C, Sweatt JD. 1999. The mitogen-activated protein kinase cascade couples PKA and PKC to cAMP response element binding protein phosphorylation in area CA1 of hippocampus. J Neurosci 19: 4337-4348.

Rodrigues SM, Schafe GE, Ledoux JE. 2004. Molecular mechanisms underlying emotional learning and memory in the lateral amygdala. Neuron 44: 75-91.

Schafe GE, Nadel NV, Sullivan GM, Harris A, Ledoux JE. 1999. Memory consolidation for contextual and auditory fear conditioning is dependent on protein synthesis, PKA, and MAP kinase. Learn Mem 6: 97-110.

Schafe GE, Nader K, Blair HT, Ledoux JE. 2001. Memory consolidation of Pavlovian fear conditioning: A cellular and molecular perspective. Trends Neurosci 24: 540-546.

Schafe GE, Atkins CM, Swank MW, Bauer EP, Sweatt JD, Ledoux JE. 2002. Activation of ERK/MAP kinase in the amygdala is required for memory consolidation of Pavlovian fear conditioning. J Neurosci 20: 8177-8187.

Shi C, Davis M. 1999. Pain pathways involved in fear conditioning measured with fear-potentiated startle: Lesion studies. J Neurosci 19: $420-430$

Shiflett MW, Martini RP, Mauna JC, Foster RL, Peet E, Thiels E. 2008. Cue-elicited reward-seeking requires extracellular signal-regulated kinase activation in the nucleus accumbens. J Neurosci 28: 1434-1443.

Szapiro G, Galante JM, Barros DM, Levi de Stein M, Vianna MR, Izquierdo LA, Izquierdo I, Medina JH. 2002. Molecular mechanisms of memory retrieval. Neurochem Res 27: 1491-1498.

Vertes RP. 2006. Interactions among the medial prefrontal cortex, hippocampus and midline thalamus in emotional and cognitive processing in the rat. Neuroscience 142: 1-20.

Vertes RP, Hoover WB. 2008. Projections of the paraventricular and paratenial nuclei of the dorsal midline thalamus in the rat. J Comp Neurol 508: 212-237.

Wright CI, Groenewegen HJ. 1995. Patterns of convergence and segregation in the medial nucleus accumbens of the rat: Relationships of prefrontal cortical, midline thalamic, and basal amygdaloid afferents. J Comp Neurol 361: 383-403.

Received November 22, 2012; accepted in revised form January 18, 2013. 


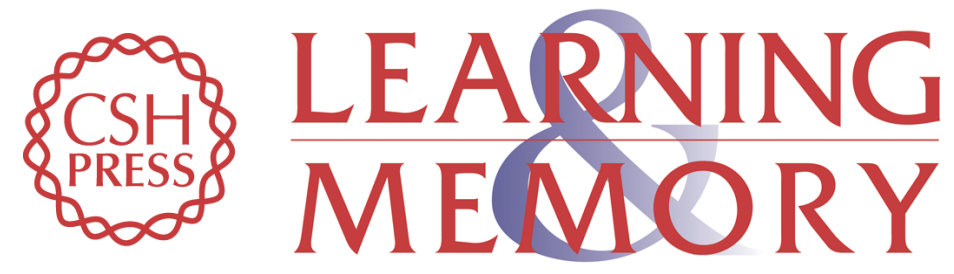

\section{Neural correlates of appetitive-aversive interactions in Pavlovian fear conditioning}

Helen M. Nasser and Gavan P. McNally

Learn. Mem. 2013, 20:

Access the most recent version at doi:10.1101/Im.029744.112

References This article cites 47 articles, 9 of which can be accessed free at:

http://learnmem.cshlp.org/content/20/4/220.full.html\#ref-list-1

License

Email Alerting Receive free email alerts when new articles cite this article - sign up in the box at the Service top right corner of the article or click here. 